\title{
Simulation of Reflectance of Porous Thin-Layer Systems
}

\author{
A. Huet \\ Departamento de \\ Nanotecnología, Centro de \\ Física Aplicada y Tecnología \\ Avanzada \\ Universidad Nacional \\ Autónoma de México, México.
}

\author{
C. F. Ramirez-Gutierrez \\ Posgrado en Ciencia e \\ Ingeniería de Materiales, \\ Centro de Física Aplicada y \\ Tecnología Avanzada \\ Universidad Nacional \\ Autónoma de México, México.
}

\author{
M. E. Rodriguez-García \\ Departamento de \\ Nanotecnología, Centro de \\ Física Aplicada y Tecnología \\ Avanzada \\ Universidad Nacional \\ Autónoma de México, México.
}

\begin{abstract}
An important class of optical devices consists of multiple thin layers of porous materials such as silicon. After producing samples, one often has limited optical data, for example, reflectance spectrum and under this limitation one must fit the data to specific optical models in order to obtain the complete optical functions. This process may lead to unphysical fits if supplementary knowledge of the reflectance signal is not applied. For this reason, the simulation and modelling of the optical properties of this type of structures is an important tool for the design of optical devices. In this paper we use simulations to identify some key aspects of how reflectance is affected by different physical parameters that are carried by commonly used models in order to provide a basic guideline for analyzing and fitting the reflectance signal. We demonstrate some general trends for the reflectance of porous systems under low porosity and high porosity regimes analyzing its dependence on layer-thickness, energy gap and surface roughness. We emphasize trends that are independent of which model is used to represent the porosity.
\end{abstract}

\section{General Terms}

Simulation of reflectance for porous materials.

\section{Keywords}

Optics, reflectance, porous materials, thin-layer systems.

\section{INTRODUCTION}

Thin layers of porous silicon (PS) are relatively easy and inexpensive to obtain from $\mathrm{p}$ and $\mathrm{n}$-type silicon wafers. This material presents many interesting physical properties and has a wide range of applications $[1,2,3]$. In particular, PS can be fabricated to achieve a specific optical performance as demanded by each specific application [4, 5]. Several parameters may affect the optical properties of a thin PS layer, namely: thickness, porosity (fraction), size of pores, topology of the porous structure, doping level and oxidation, among others. The nature of the fabrication process usually results on a considerable variability on the parameters that are relevant to optics and it is often necessary to adjust multiparametric optical models to limited experimental data. In this process, usually the simplest optical measurement to obtain is the reflectance for which one must find a proper fit. As the number of parameters to fit increases, it can be cumbersome to perform the adjustments without some experience on how the reflectance behaves in response to the model parameters or sample variability. Another problem is that the algorithms that find the best fit to data will often find just one of multiple solutions, the "best fit" obtained may be numerically better but it may contain unphysical values of the parameters. Such problems can be avoided by providing a physically reasonable initial guess to the fitting algorithm. But making a sensible guess requires one to know the basics of the behavior of reflectance under the variation of the usual parameters.

In this paper we provide a guideline for the behavior of the reflectance signal in thin porous layer systems. We work with a simulated substrate using the model by Forouhi and Bloomer [6]. In this model the complex dielectric function of the material is derived from a one-electron model using a finite lifetime for electron states, producing good agreement for a variety of materials. We work with the parameters they reported for silicon.

To simulate different configurations we must be able to vary sample parameters such as layer thickness or introduce multiple layers the optical functions fur such configurations may be obtained from different summation techniques. For our simulation we use two different methods: the LTR method and the Generalized Matrix Method [7, 8].

The porosity is simulated and compared using two different effective medium theories: the Bruggeman and the Looyenga $[9,10,11,12]$. These models are known to be fundamentally different and their best performance is achieved on different porosity regimes.

In section 2, we simulate a system composed of a thin porous layer on top of semi-infinite substrate. We analyze how the reflectance is affected by variations of the porous layer thickness at different porosity regime, we emphasize a trend that is independent of the porosity model. Next, we study the response of the reflectance as the porosity increases. We show how the reflectance changes its trend after a critical value of the porosity. Once more we show that the main trend is independent of the porosity model.

The porous structure of a material can have important effects on the electron states, affecting the optical functions $[3,13]$. In section 3 , we study the change in reflectance on the $\mathrm{UV}-\mathrm{V}$ is region under a variation of the energy gap outside this interval. We address the different trends obtained depending on the porosity regime.

The presence of surface roughness at the interfaces of a stratified system has an effect on the reflectance signal and one must account for it in order to obtain better fits. In section 4 , we simulate the influence of roughness using the method proposed by Mitsas and Siapkas [8]. We analyze the variation of reflectance when the roughness is present at three different types of interfaces: porous medium-air, porous mediumsubstrate, and between two porous layers. We find general trends for reflectance for each of these cases.

\section{SIMULATING THE LAYER THICKNESS AND POROSITY}

Consider a system composed of one thin porous layer on top of substrate. The reflectance spectrum of this type of system shifts according to the thickness of the top layer. For example, 
in the case of porous silicon the layer thickness may range from about $10 \mathrm{~nm}$ to $100 \mathrm{~nm}$ and depending on the fabrication process, one can expect to obtain considerable variability in the samples. As an example, we have simulated the reflectance of one thin porous silicon layer on top of silicon substrate. The porosity has been introduced using the Bruggeman model:

$$
p \frac{\varepsilon_{M}-\varepsilon_{e f f}}{\varepsilon_{M}+2 \varepsilon_{e f f}}+(1-p) \frac{\varepsilon-\varepsilon_{e f f}}{\varepsilon+2 \varepsilon_{e f f}}=0
$$

Where $p$ is the porosity and $\varepsilon_{M}, \varepsilon$ and $\varepsilon_{e f f}$ are the complex dielectric constants of the substrate, vacuum and the porous medium respectively. We have simulated the effect of varying the layer thickness from $25 \mathrm{~nm}$ to $45 \mathrm{~nm}$ and have used a low value for the porosity: $p=0.2$. The simulation shows that small changes in e thickness do not affect the characteristic reflectance peaks of the substrate, instead, their effect is to attenuate the falling rate of reflectance to the right of the main peaks while remaining steady on the left. On the large wavelength region of the spectrum the reflectance shifts upwards, see Figure 1.

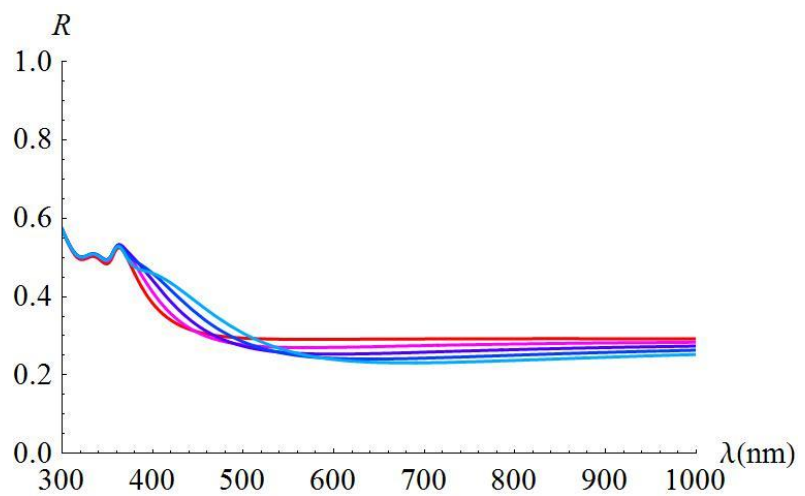

Figure 1: The plot shows the change in reflectance as the thickness of the porous layer is increased from $25 \mathrm{~nm}$ (red) to $45 \mathrm{~nm}$ (light blue). The porosity is set at $p=0.2$. The Bruggeman model is used.

However, when the porosity is high, varying the layer thickness has a clear effect on the reflectance peaks (substrate). In Figure 2 a porosity value of $p=0.8$ has been set, the effect of changing the thickness is now more important at shorter wavelengths.

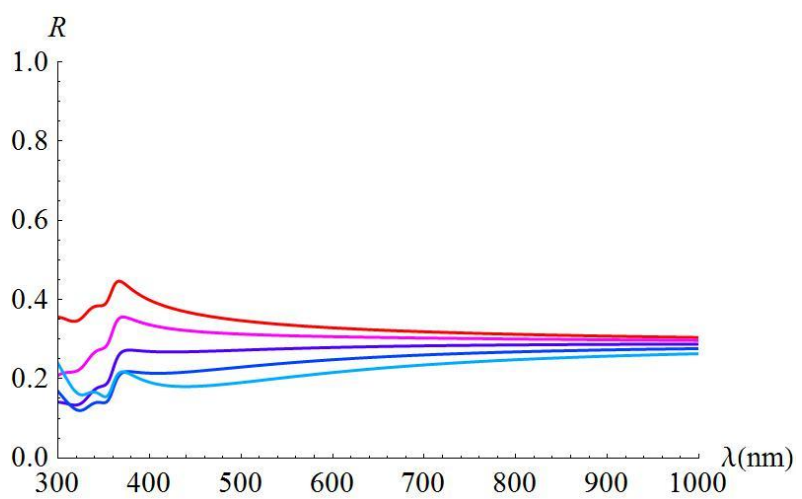

Figure 2: The plot shows the change in reflectance as the thickness of the porous layer is increased from $25 \mathrm{~nm}$ (red) to $45 \mathrm{~nm}$ (light blue). The porosity is set at $p=0.8$. The Bruggeman model is used.

It is important to mention that the change in trend observed between low porosity and high porosity is independent of the effective medium model used. In the Looyenga model the porosity is given by:

$$
\varepsilon_{e f f}{ }^{1 / 3}=(1-p) \varepsilon^{1 / 3}+p \varepsilon_{M}{ }^{1 / 3}
$$

This model is fundamentally different as it accounts for nonzero percolation strength at any porosity. It is expected to work well at high porosities [8]. In Figure 3 we simulate the same system as in figures 1 and 2 but at this time, we use the Looyenga model, the same trends are obtained.
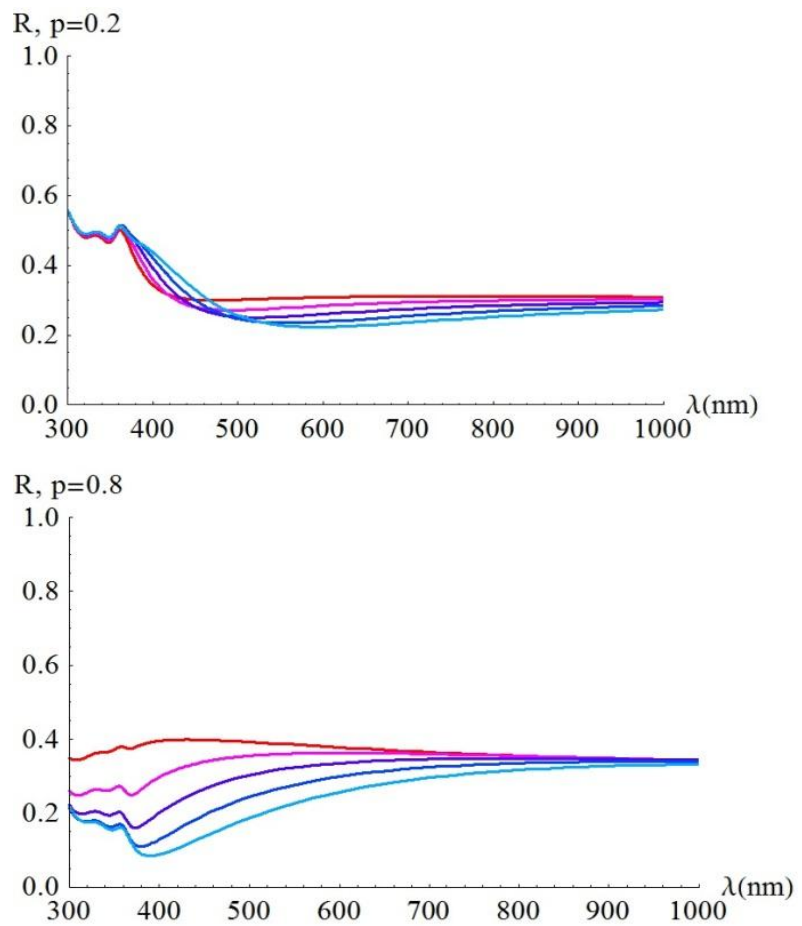

Figure 3: The plots show the change in reflectance as the thickness of the porous layer is increased from $25 \mathrm{~nm}$ (red) to $45 \mathrm{~nm}$ (light blue). For both low porosity $(p=0.2)$ and high porosity $(\mathrm{p}=0.8)$. The Looyenga model is used.

Varying the thickness on a wider range causes new local extreme values to appear due to interference. The example presented shows how the $\mathrm{p}=0.2$ system behaves when the thickness varies from $40 \mathrm{~nm}$ to $440 \mathrm{~nm}$. For the larger thickness we obtain two new local maxima. Naturally, this trend is also independent of the porosity model.

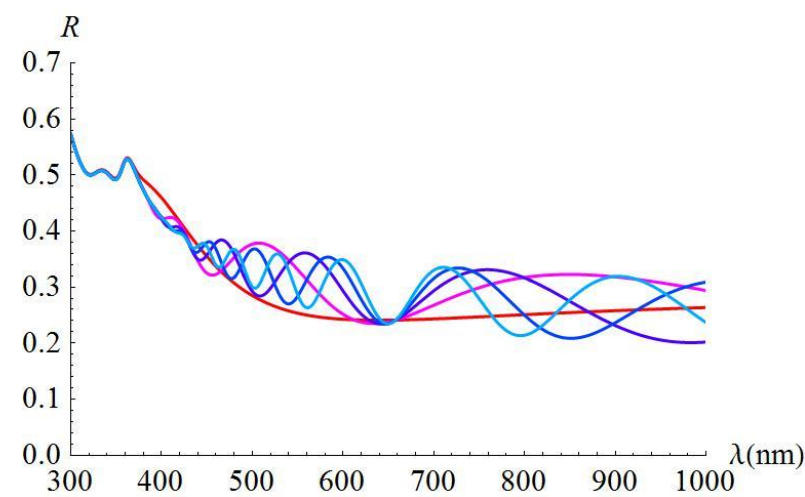

Figure 4: The plot shows the appearance new local maxima of reflectance as a result of increasing the layer thickness on a wider range, from 40 (red), 140, 240, 340 and $440 \mathrm{~nm}$ (blue). The porosity was set at $p=0.2$. The Bruggeman model was used. 
When the thickness is fixed but the porosity is varied the reflectance does not follow a monotonous trend, in fact, there is a critical value of the porosity beyond which the reflectance changes its trend. One could expect this value to be $\mathrm{p}=0.5$ but it is not always the case. The simulation in Fig. 5 (top) shows a $50 \mathrm{~nm}$ layer on top of substrate, the porosity is varied from $p=0.1$ up to $p=0.6$ the behavior of the reflectance as porosity increases is monotonous, it is shifting down and a point of zero reflectance appears for $\mathrm{p}=0.6$, after this value the reflectance changes its trend. In Figure 5 (bottom) the same system is shown but now the porosity starts at $\mathrm{p}=0.6$ and goes up to $\mathrm{p}=0.9$, the trend has changed; now the reflectance is shifting upwards as the porosity increases. This feature also helps to understand what was shown in Figures 1 and 2 (or 3), i.e. that the effect of changing the layer thickness is different depending on whether the value of porosity is higher or lower than the critical value.

This change of trend after a critical value of porosity is also model independent and it happens also for the Looyenga model. However, we note one difference: with the Looyenga model reflectance does not drop to zero for any wavelength near the critical porosity, this model performs better at high porosities.

\section{Low porosities}

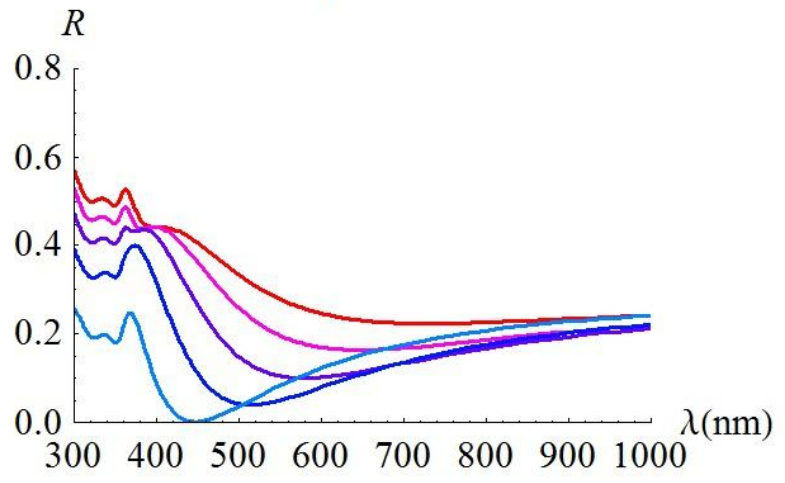

\section{High porosities}

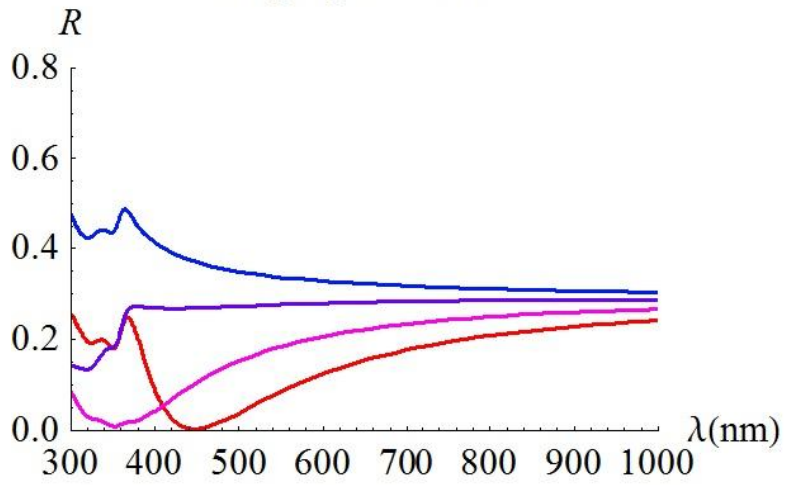

Figure 5: The plots show the change of reflectance as the porosity is increased. On top the porosity runs from $p=0.2$ (red) up to $p=0.6$ (light blue), the reflectance is shifts down until a critical porosity is reached. On the bottom the porosity runs from $\mathrm{p}=0.6$ (red) to $\mathrm{p}=0.9$ (blue), showing a different trend. The thickness of the porous layer was set at $50 \mathrm{~nm}$ (Bruggeman model).

\section{SIMULATING THE OPTICAL FUNCTIONS OF THE BULK MATERIAL}

In this section, we analyze how the reflectance shifts due to a variation of the optical properties of the material itself. An important effect in porous silicon is the shift of the band gap with respect to the bulk material due to confinement effects $[3,12]$. It is useful to understand the trend that such shifts produce in the reflectance over other wavelength regimes far from the gap, such as the UV-Vis region. For this matter, we simulate the optical functions of silicon using the model by Forouhi and Bloomer which explicitly includes the energy gap as parameter and for which accurate fits exist for many materials including silicon [11]. As before, we find that this effect is coupled to the porosity value and thus, it must be analyzed over the full porosity range.

The reflectance is simulated for a starting band gap of 1107 $\mathrm{nm}$ and its value is shifted down to $732 \mathrm{~nm}$. As before, the reflectance has a different response depending on the porosity. For low porosity $\mathrm{p}=0.2$, the shift in the band gap decreases the reflectance in all the spectrum except for a small transition region where we pass from the characteristic peaks into the region of monotonous optical behavior. See Figure 7.

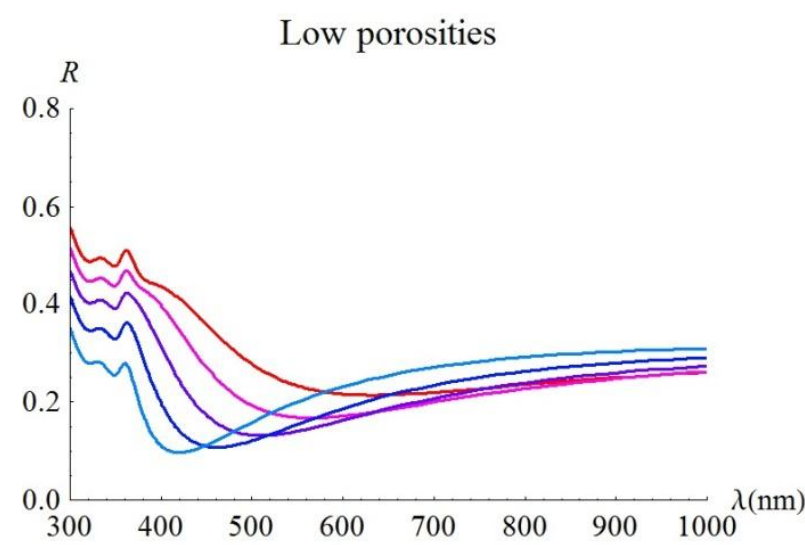

High porosities

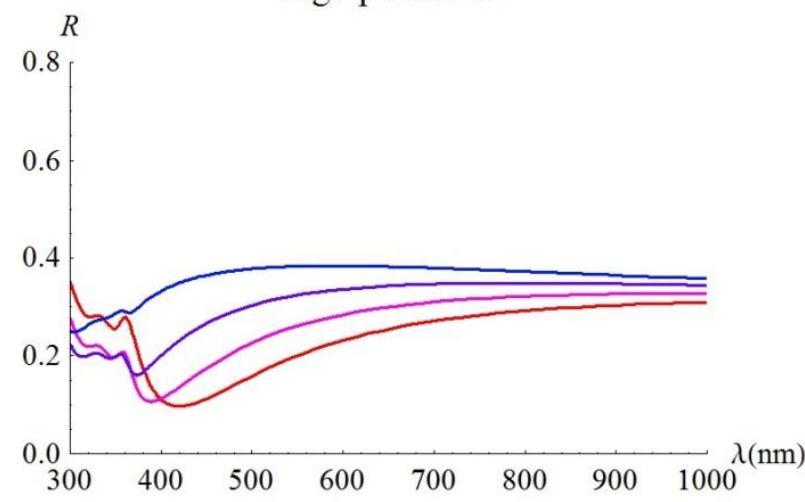

Figure 6: The plots show the change of reflectance as the porosity is increased. On top the porosity runs from $p=0.2$ (red) up to $\mathrm{p}=0.6$ (light blue), the reflectance is shifts down until a critical porosity is reached. On the bottom the porosity runs from $p=0.6$ (red) to $p=0.9$ (blue), showing a different trend. The thickness of the porous layer was set at $50 \mathrm{~nm}$ (Looyenga model). 


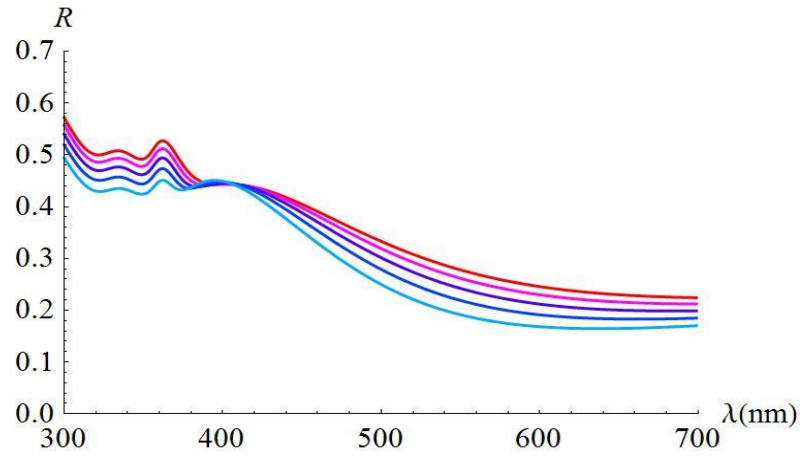

Figure 7: The porosity is set at $\mathbf{p}=0.2$ using the Bruggeman model. The reflectance is simulated for a starting band gap of $1107 \mathrm{~nm}$ (red) and its value runs down to $732 \mathrm{~nm}$ (blue). The reflectance gets reduced almost everywhere in the optical range.

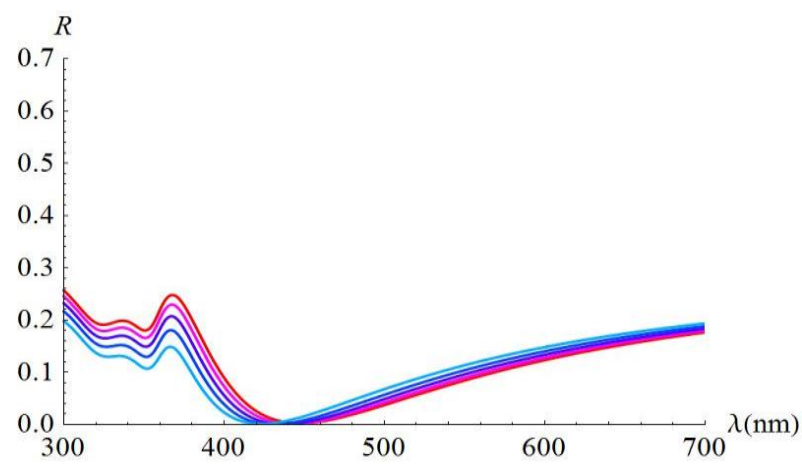

Figure 8: The porosity is set at $\mathbf{p}=\mathbf{0 . 6}$ using the Bruggeman model. The reflectance is simulated for a starting band gap of $1107 \mathrm{~nm}$ (red) and its value runs down to $732 \mathrm{~nm}$ (blue). The reflectance gets reduced towards the UV region but increased towards the infrared region.

There is also a change in trend that happens at a critical porosity, when the porosity reaches $\mathrm{p}=0.6$, the shift in the energy gap still lowers the reflectance in the UV region but now the reflectance for large wavelengths is increasing (see Figure 8).

For the same system we simulate the high porosity regime, the shift on the energy gap has smaller but opposite effect compared to the low porosity cases, to be specific, it increases the reflectance. Also, its main effect happens at shorter wavelengths. See Figure 9.

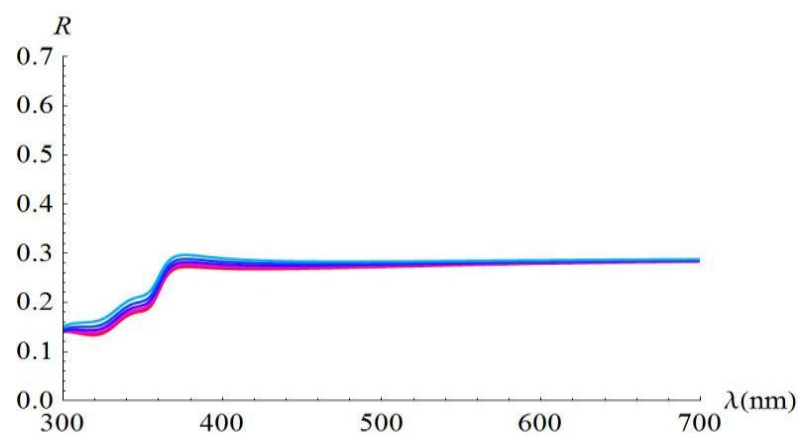

Figure 9: The porosity is set at $\mathrm{p}=0.8$ using the Bruggeman model. The reflectance is simulated for a starting band gap of $1107 \mathrm{~nm}$ (red) and its value runs down to $732 \mathrm{~nm}$ (blue). The reflectance increases everywhere in the optical range with a stronger shift at smaller wavelengths.

\section{SIMULATING SURFACE ROUGHNESS}

Systems composed of a single or multiple layers on top substrate always present some degree of surface roughness in each interface, for example between the porous medium and air, or between separate layers of the porous medium. This surface roughness has an effect on the reflectance spectrum due to incoherence [8]. Some simple general trends can be identified depending on which interface presents roughness.

To introduce incoherence and partial incoherence in our simulation, a transfer matrix has been used. In this model, each interface is characterized by refractive matrix $\mathrm{Wi}-1, \mathrm{i}$ and propagation matrix Ui which contains the optical constants and thickness of ith layer [14].

These matrices are

$$
\mathbf{W}_{i-1, i}=\frac{1}{t_{R i}}\left(\begin{array}{cc}
1 & -r_{L i} \\
r_{R i} & t_{R i} t_{L i}-r_{R i} r_{L i}
\end{array}\right)
$$

and

$$
\mathbf{U}_{i}=\left(\begin{array}{cc}
\exp \left(-i \varphi_{i}\right) & 0 \\
0 & \exp \left(i \varphi_{i}\right)
\end{array}\right)
$$

where $\varphi_{i}=2 \pi / \lambda \widetilde{n_{i}} d_{i}$ is the phase difference, and $r_{L i}$ and $r_{R i}$ are the usual left and right Fresnel coefficients. The resulting transfer matrix is given by

$$
\mathbf{T}=\mathbf{W}_{01} \mathbf{U}_{1} \mathbf{W}_{12} \mathbf{U}_{2} \ldots \mathbf{W}_{m, m+1}=\left(\begin{array}{ll}
s_{11} & s_{12} \\
s_{21} & s_{22}
\end{array}\right)
$$

where the reflection coefficient of the stratified system is

$$
r=\frac{s_{21}}{s_{11}}
$$

The random roughness of each interface is represented by the following modified Fresnel coefficients

$$
\begin{aligned}
& r_{R i}^{\prime}=r_{R i} \exp \left[-2\left(2 \pi \sigma_{i} \hat{n}_{i-1} / \lambda\right)^{2}\right]=\alpha r_{R i} \\
& r_{L i}^{\prime}=r_{L i} \exp \left[-2\left(2 \pi \sigma_{i} \hat{n}_{i} / \lambda\right)^{2}\right]=\beta r_{L i} \\
& t_{R i}^{\prime}=t_{R i} \exp \left[-1 / 2\left(2 \pi \sigma_{i} / \lambda\right)^{2}\left(\hat{n}_{i}-\hat{n}_{i-1}\right)^{2}\right]=\gamma t_{R i} \\
& \mathrm{t}_{\mathrm{Li}}^{\prime}=\mathrm{t}_{\mathrm{Li}} \exp \left[-1 / 2\left(2 \pi \sigma_{\mathrm{i}} / \lambda\right)^{2}\left(\hat{\mathrm{n}}_{\mathrm{i}-1}-\hat{\mathrm{n}}_{i}\right)^{2}\right]=\gamma \mathrm{t}_{\mathrm{Li}}
\end{aligned}
$$

Using this methodology, we can introduce a RMS roughness $\sigma$ in any interface but it is important to note that this method assumes that the value of $\sigma$ is very small compared to the minimum wavelength in the reflectance measurements.

First, we consider the presence of roughness in the interface between the porous medium and air. This is the simplest case as the roughness tends to decrease the reflectance over the entire spectrum: However, the decrease in reflectance is not the same for all wavelengths, as it is shown in Figure 10 where we observe a more important effect towards the UV region of the spectrum.

Systems of either one or multiple layers produce local maxima and minima in the reflectance due to interference. If we add roughness at the interface between substrate and porous medium, we observe a different trend: as the roughness increases the interference pattern gets washed out and the reflectance plot is "flattened", meaning that the reflectance peaks shift down while the local minima shift up. We also note that, in this case, roughness has no effect on the characteristic reflectance peaks of the substrate since those are not derived from an interference effect (see Figure 11). 


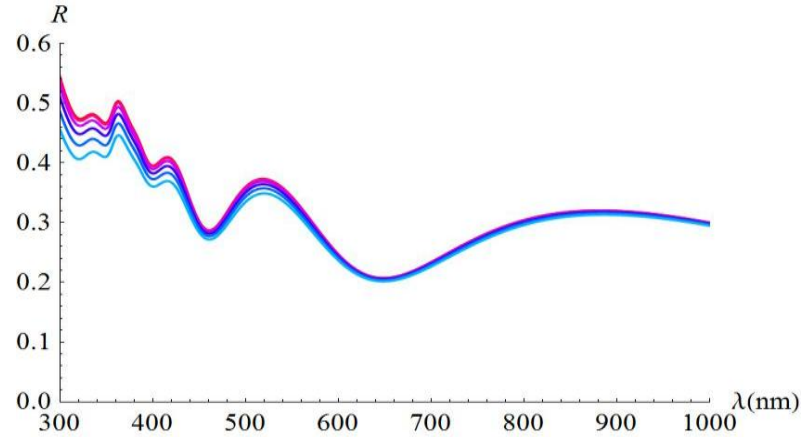

Figure 10: The plot shows the reflectance of a single porous layer on top of substrate with a rough interface between porous medium and air. The substrate thickness is set at $150 \mathrm{~nm}$ with $p=0.2$ simulated with the Bruggeman model. The roughness parameter goes from $Z=0 \mathrm{~nm}$ up to $\mathrm{Z}=\mathbf{1 0} \mathbf{n m}$.

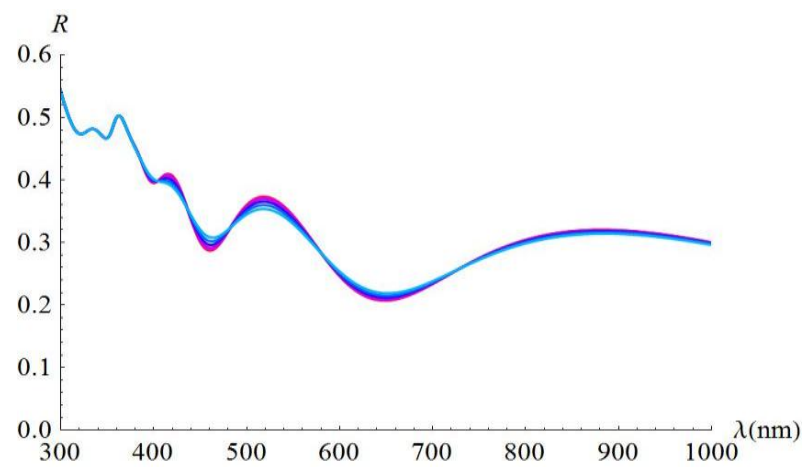

Figure 11: The plot shows the reflectance of a single porous layer on top of substrate with a rough interface between porous medium and substrate. The substrate thickness is set at $150 \mathrm{~nm}$ with $\mathrm{p}=0.2$ simulated with the Bruggeman model. The roughness parameter goes from $\mathrm{Z}=\mathbf{0} \mathrm{nm}$ up to $\mathrm{Z}=10 \mathrm{~nm}$.

Roughness may also be present at the interface between two different layers of the same porous medium. Each layer may have a different porosity value. In this case, as the roughness increases one finds a trend that is similar to that of Figure 11, that is, the reflectance extreme values in the interference region get flattened provided that the two porous layers are not too different from each other (see Figure 12).

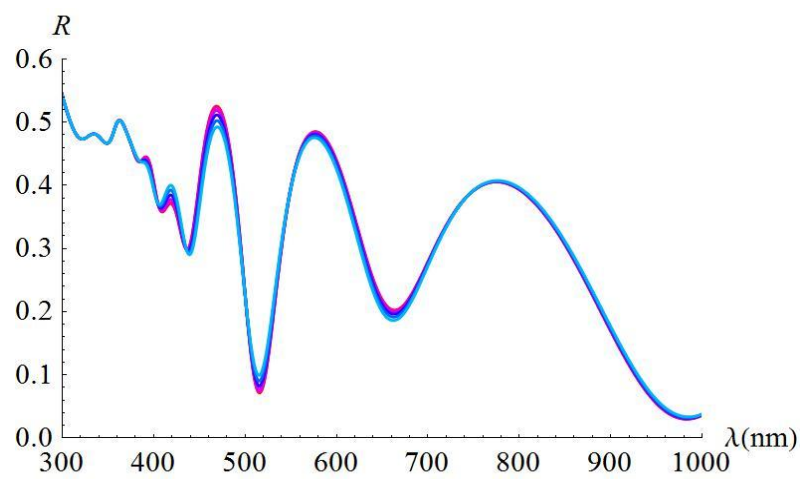

Figure 12: The plot shows the reflectance of two porous layers on top of substrate. A rough interface between the porous layers has been simulated. The substrate thickness is set at $150 \mathrm{~nm}$ with $p=0.2$ for the outer layer and $p=0.5$ for the inner layer (Bruggeman model). The roughness parameter goes from $Z=0 \mathrm{~nm}$ up to $Z=10 \mathrm{~nm}$.

\section{CONCLUSIONS}

For a wide variety of systems it is possible to identify trends that help as a guideline for a better understanding of the reflectance spectrum and more physically accurate fit of the experimental data using different optical models. Systems with low porosity and high porosity often present different reflectance trends while varying the same parameter. It is helpful identify the optically critical value of the porosity that separates the low porosity from the high porosity regime. Even though, there are different optical models to account for porosity, one is able to identify important aspects of the reflectance spectrum that are independent of the model. We have provided a simple guideline to understand the effect of relevant parameters that determine reflectance such as layer thickness, porosity, energy gap and interface roughness.

In the future, we wish to extend the present work to simulate other variables that affect the reflectance, such as, the presence of oxide and variations in the carrier density of the substrate. We also expect to apply our methods of reflectance analysis to practical devices such as Bragg reflectors and Fabry-Pérot cavities.

\section{ACKNOWLEDGMENTS}

Our thanks to PAPIIT for supporting project number IN115113. A. Huet and C. F. Ramirez-Gutierrez received support from CONACYT.

\section{REFERENCES}

[1] Canham, L. T. 1990. Silicon Quantum Wire Array Fabrication by Electrochemical and Chemical Dissolution of Wafers. Appl. Phys. Lett., Vol 57, 1046.

[2] Canham, L. T. 1997. Properties of Porous Silicon. INSPEC, London, 1st Ed.

[3] Cullis, A. G., Canham, L. T., and Calcott P. D. J. 1997. The Structural and Luminescence Properties of Porous Silicon, J. Appl. Physics, Vol 82, No 3.

[4] Patel, P. N. and Mishra, V. 2012. Simulations and Analysis of Nano Scale Porous Silicon Structures for Optical Sensor Applications. International Journal of Computer Applications, Vol 56, No10, 0975-8887.

[5] Patel, P. N., Mishra, V., and Panchal, A. K. 2012. Theoretical and Experimental Study of Nano Porous Silicon Photonic Microcavity Optical Sensor Devices. Adv. Nat. Sci.: Nanosci. Nanotechnol. Vol. 3.

[6] Forouhi, A. R. and Bloomer, I. 1988. Optical Properties of Crystalline Semiconductors and Dielectrics. Phys. Rev. B, Vol 38, No 3, 1865-1874.

[7] Mazilu, M., Miller, A., and Donchev, V. T. 2001 Modular Method for Calculation of Transmission and Reflection in Multilayered Structures, Appl. Opt. 40, 6670-6676.

[8] Mitsas, C. L. and Siapkas, D. I. 1995. Generalized Matrix Method for Analysis of Coherent and Incoherent Reflectance and Transmittance of Multilayer Structures With Rough Surfaces, Interfaces, And Finite Substrates. Appl. Opt., Vol 34, No 10.

[9] Bruggeman, D. A. G. 1935. Berechnung Verschiedener Physikalischer Konstanten von Heterogenen Substanzen. Ann. Phys. 24, 636-679. 
[10] Theiss, W. 1997. Optical Properties of Porous Silicon. Surface Science Reports, Vol 29, Issues 3-4, 91-93, 95192.

[11] Theiss, W., Henkel, S., and Arntzen, M. 1995. Connecting Microscopic and Macroscopic Properties of Porous Media: Choosing Appropriate Effective Medium Concepts. Thin Solid Films, 255, 177-180.

[12] Theiss, W. 1996. The Dielectric Function of Porous Silicon - How to Obtain It and How to Use It, Thin Solid Films, 276, 7-12.
[13] Delley, B. and Steigmeier, E. F. 1995. Size Dependence of Band Gaps in Silicon Nanostructures. Appl. Phys. Lett. Vol 67, 2370.

[14] Katsidis, C. C. and Siapkas, D. I. 2002. General Transfer-Matrix Method for Optical Multilayer Systems with Coherent, Partially Coherent, and Incoherent Interference. Appl. Opt. 41, 3978-3987. 